\title{
Computational model of 18650 lithium-ion battery with coupled strain rate and SOC dependencies
}

Article in Applied Energy · June 2016

DOI: 10.1016/j.apenergy.2016.03.108

CITATIONS

12

4 authors:

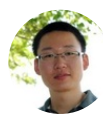

\section{Jun Xu}

Beihang University (BUAA)

62 PUBLICATIONS 344 CITATIONS

SEE PROFILE

2. Xinyi Wang

Beijing Forestry University

1 PUBLICATION 12 CITATIONS

SEE PROFILE
READS

173
Liu Binghe

Beihang University (BUAA)

9 PUBLICATIONS 36 CITATIONS

SEE PROFILE

\section{Hu Dayong}

Beihang University (BUAA)

21 PUBLICATIONS 105 CITATIONS

SEE PROFILE

Some of the authors of this publication are also working on these related projects:

Project
Multi-scale mechanical-electrochemical-thermal coupled modeling framework for lithium-ion battery under mechanical abuse View project

National Natural Science Foundation of China View project 


\title{
Computational model of 18650 lithium-ion battery with coupled strain rate and SOC dependencies
}

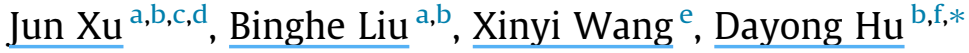 \\ ${ }^{a}$ Department of Automotive Engineering, School of Transportation Science and Engineering, Beihang University, Beijing 100191, China \\ ${ }^{\mathrm{b}}$ Advanced Vehicle Research Center (AVRC), Beihang University, Beijing 100191, China \\ ' State Key Laboratory of Nonlinear Mechanics (LNM), Institute of Mechanics, Chinese Academy of Sciences, Beijing 100190, China \\ ${ }^{\mathrm{d}}$ State Key Laboratory for Strength and Vibration of Mechanical Structures, School of Aerospace Engineering, Xi'an Jiaotong University, Xi'an 710049, China \\ e School of Engineering, Beijing Forestry University, Beijing 100083, China \\ f Department of Aircraft Airworthiness Engineering, School of Transportation Science and Engineering, Beihang University, Beijing 100191, China
}

\section{H I G H L I G H T S}

- An anisotropic model to describe mechanical behaviors of LIB is established.

- SOC dependency is included in the mechanical model of the jellyroll.

- Dynamic effect is considered in the model for LIB.
G R A P H I C A L A B S T R A C T
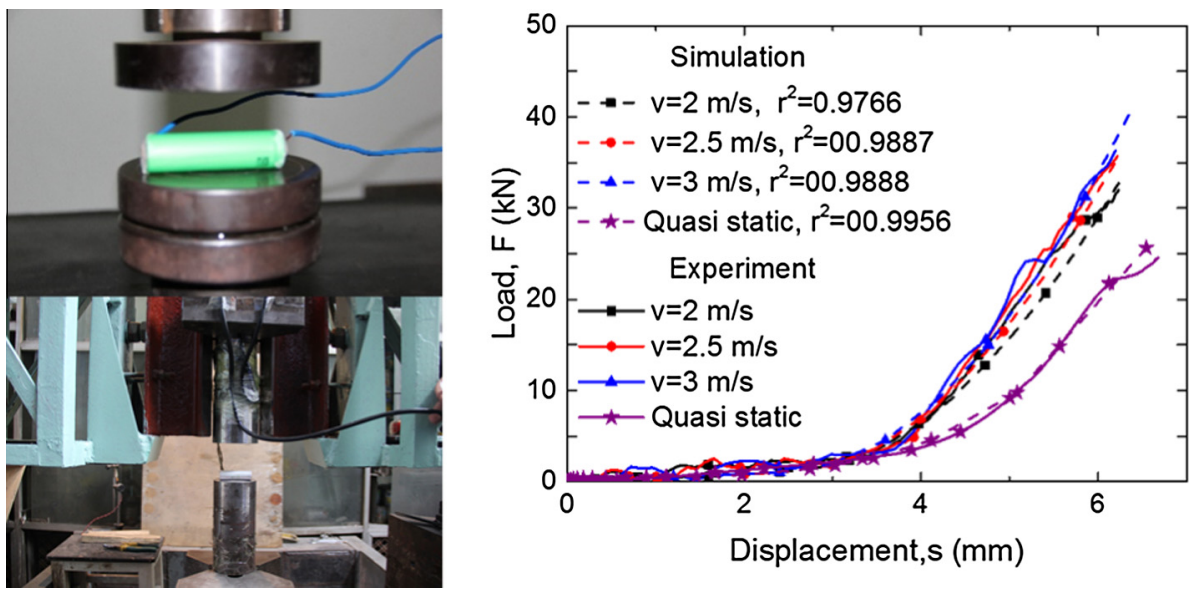

\section{A B S T R A C T}

Highly nonlinear structures and constituent materials and hazardous experiment situations have resulted in a pressing need for a numerical mechanical model for lithium-ion battery (LIB). However, such a model is still not well established. In this paper, an anisotropic homogeneous model describing the jellyroll and the battery shell is established and validated through compression, indentation, and bending tests at quasi-static loadings. In this model, state-of-charge (SOC) dependency of the LIB is further included through an analogy with the strain-rate effect. Moreover, with consideration of the inertia and strainrate effects, the anisotropic homogeneous model is extended into the dynamic regime and proven capable of predicting the dynamic response of the LIB using the drop-weight test. The established model may help to predict extreme cases with high SOCs and crashing speeds with an over $135 \%$ improved accuracy compared to traditional models. The established coupled strain rate and SOC dependencies of the numerical mechanical model for the LIB aims to provide a solid step toward unraveling and quantifying the complicated problems for research on LIB mechanical integrity.

(c) 2016 Elsevier Ltd. All rights reserved.

\footnotetext{
* Corresponding author at: Advanced Vehicle Research Center (AVRC), Beihang University, Beijing 100191, China.

E-mail address: hudayong@buaa.edu.cn (D. Hu).
} 


\section{Introduction}

Lithium-ion batteries (LIBs) are increasingly widely used in modern industrial products, e.g., unmanned aircraft, vehicles, robots, and cellphones, among others [1-7]. Take the electric vehicle (EV) for example. In 2013, the annual sales of EVs reached 505,000 in the US, 904,000 in Japan, and 22,000 in Europe [8]. Such large quantities of vehicles may inevitably suffer from crash accidents and experience frequent foreign object intrusion/impact on the LIB package during driving. Thus, the mechanical integrity of LIBs has attracted considerable research with respect to LIB safety, where the failure of a battery depends on chemomechanical type [9], as well its transient and long-term electrochemical performance.

Previous pioneering work focused on the individual components properties including electrodes [10] and separator [9,11,12] and the general response of the LIB subject to various mechanical loadings, such as radial crushing, bending, and indentation. From the structural point of view, jellyroll and battery shell are the two core structural parts of the entire LIB. In the battery cell level, bending, indentation, and radial crushing $[13,14]$ tests were proposed to study the overall mechanical response subject to loadings, where jellyroll is regarded as a macroscopic homogeneous model. Constitutive models to predict the radial direction properties of jellyroll from the flow rule and the conception of volumetric strain were proposed by Greve and Fehrenbach [13] and Wierzbicki and Sahraei [15] respectively. Then the representative volume element model of the jellyroll was identified $[16,17]$. The anisotropic property of the structure as a result of geometry and material was not considered by these two models. Therefore, these models may accurately predict radial crushing and indentation tests of the jellyroll, but fail for the bending cases.

Jellyroll consists of separator, cathode, anode, active particles, and adhesives to bond active particles on the electrodes [18]. Ruling out the structural effects makes obtaining the material parameters of cathode and anode by tension and compression tests through staking up layers of the material rather straightforward $[16,17]$. The separator plays an important role in possible internal short-circuit and long-term electrochemical degradation [19-23]. Numerous efforts have been exerted to study the mechanical properties of the separator through carefully designed tension, compression tests with the possible large deformation, and highly anisotropic and obvious viscoelasticity exhibited during experiments [22,24-26]. In addition, nanoindentation tests were proposed to study the mechanical properties of active particles at nanoscales $[27,28]$.

However, current available finite element models of LIB are not capable of predicting the mechanical behavior subject to bending, where the most possible load the cell has in vehicle crashing occurs in the radius direction bending [13]. Furthermore, dynamic loading, which in essence is the bottleneck in studying the LIB mechanical integrity at crash scenarios, cannot be well represented. Xu et al. made the first step to experimentally study the dynamic behavior of the jellyroll without further experiment validation [29]. To simulate the LIB status in the continuous charging and discharging process, the state-of-charge (SOC) effect should also be included in the model. Recent evidence has shown that the mechanical properties $[27,28]$ and volume [30] of the active particles will change due to SOC value changing; such finding may serve as the direct proof for the SOC dependent mechanical behavior of LIB.

The coating for cathode and anode materials and the separator itself are highly porous and soaked in electrolyte fluid, where ions could move around $[21,26]$. Hence, modeling the jellyroll in detail with the exact contact modeling is rather complicated among each constituent. Homogeneous modeling technique is adopted in this study. Here, the anisotropic model of the jellyroll is built to predict the various radial loading conditions to the jellyroll. After validation of the numerical model, the SOC effect is considered in this quasi-static model. Furthermore, the dynamic model is established with the coupling of strain rate and SOC effects taken into consideration. The model may predict the dynamic response effectively in comparison with the drop-weight test results.

\section{Numerical modeling of LIB in quasi-static conditions}

\subsection{The modeling samples, experiment setups and simulation boundary conditions}

The $\mathrm{Li}_{x} \mathrm{C}_{6} / \mathrm{LiCoO}_{2}$ batteries are chosen in this study, provided by SONY. The basic information is summarized in Table 1, including the physical dimensions of the whole battery and each component, as well as the electrical performance. The charge/discharge curves are shown in Fig. 1(a). A large number of mechanical experiments have been studied for this type of battery [31], discovering that the mechanical properties are related to SOCs where the maximum contribution of SOC for the difference in force capacity can be up to $40 \%$. It is true that battery shell's mechanical behavior plays an important role in overall LIB's mechanical behavior; however, the jellyroll contributes more for bearing force than the shell, demonstrated in the comparison of Figs. 1(b) and 2(b).

Bending, indentation, and radial compression tests in quasistatic conditions are conducted based on the INSTRON 5699 platform with loading rate of $5 \mathrm{~mm} / \mathrm{min}$. The force and displacement sensors are set on the indenter. In compression tests, the indenter and the bearing are flat; in indentation tests the bearing is flat and

Table 1

The information of the 18650 LIB.

\begin{tabular}{|c|c|c|}
\hline Terms & Rating & Note \\
\hline Nominal capacity & $2250 \mathrm{~mA} \mathrm{~h}$ & Typical capacity of 0.2 ItA and discharging with a cut-off voltage of $2.5 \mathrm{~V}$ \\
\hline Rated capacity & $2150 \mathrm{~mA} \mathrm{~h}$ & (at $1 \mathrm{C}$ charging and standard test atmosphere) \\
\hline Nominal voltage & $3.7 \mathrm{~V}$ & \\
\hline Charge voltage & $4.2 \pm 0.05 \mathrm{~V}$ & \\
\hline Cut off voltage & $2.5 \mathrm{~V}$ & \\
\hline Continuous maximum charge current & $2.15 \mathrm{~A}$ & \\
\hline Continuous maximum discharge current & $10 \mathrm{~A}$ & \\
\hline Size & $\phi 18.6 \mathrm{~mm} \times 64.9 \mathrm{~mm}$ & \\
\hline Weight & $43.6 \pm 1.5 \mathrm{~g}$ & \\
\hline Anode material and thickness & $\mathrm{Li}_{x} \mathrm{C}_{6} / 0.165 \mathrm{~mm}$ & \\
\hline Cathode material and thickness & $\mathrm{LiCoO}_{2} / 0.159 \mathrm{~mm}$ & \\
\hline Separator & $\mathrm{PP} / 0.018 \mathrm{~mm}$ & \\
\hline Positive current collector and thickness & $\mathrm{Al} / 0.019 \mathrm{~mm}$ & \\
\hline Negative current collector and thickness & $\mathrm{Cu} / 0.013 \mathrm{~mm}$ & \\
\hline
\end{tabular}




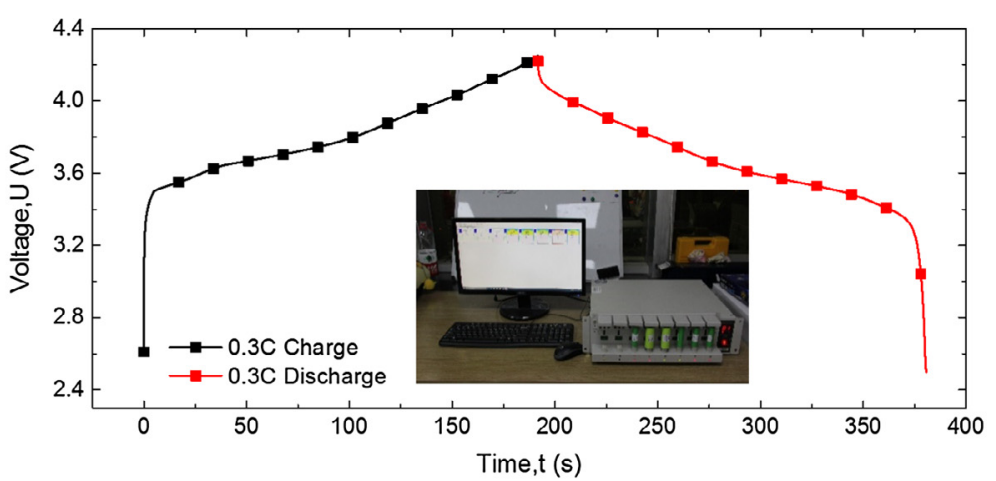

(a)

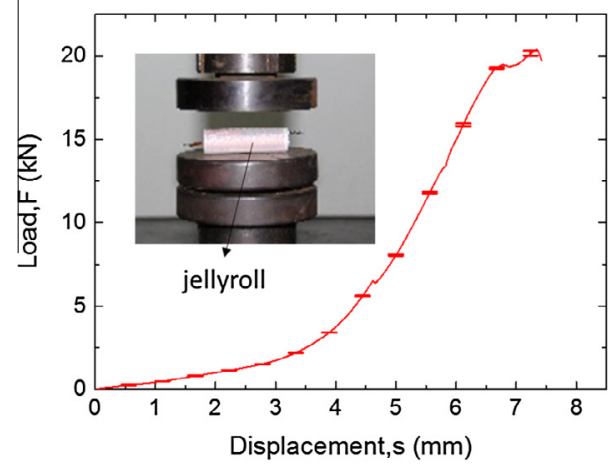

(b)

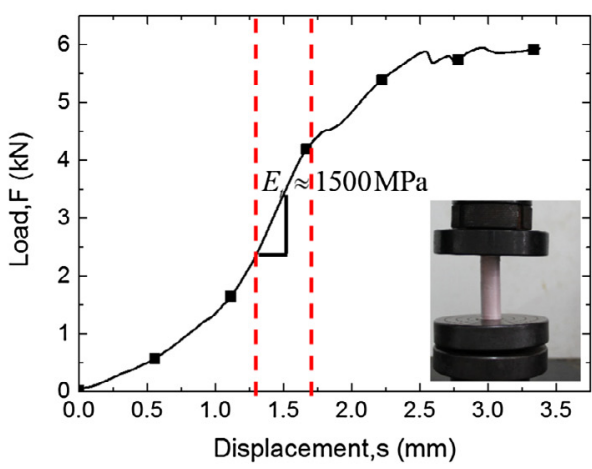

(d)

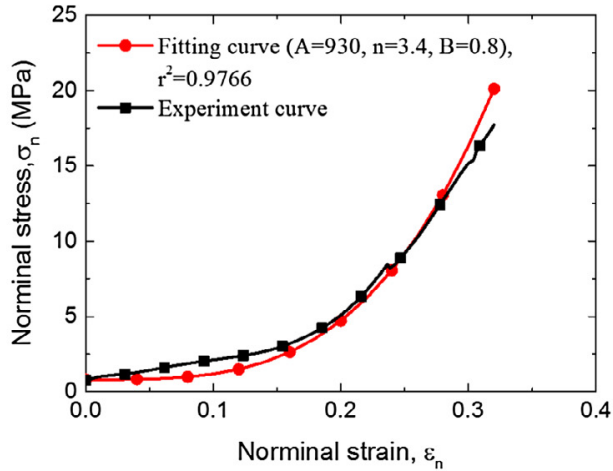

(c)

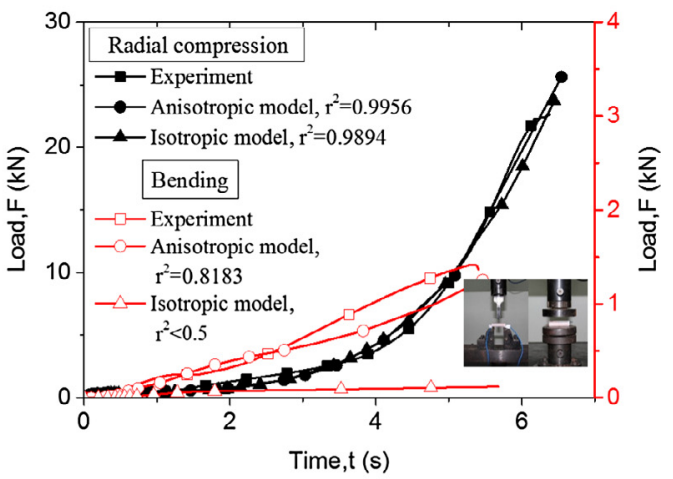

(e)

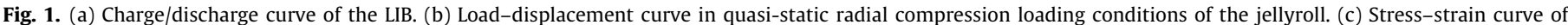

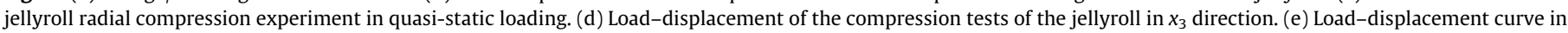
quasi-static radial compression and bending loading condition of the jellyroll for experiment and simulation.

the indenter is cylinder with radial of $10 \mathrm{~mm}$; in bending tests the bearing are two cylinders with radial of $10 \mathrm{~mm}$ with distance of $60 \mathrm{~mm}$ and the indenter is also cylinder with radial of $10 \mathrm{~mm}$.

ABAQUS/Standard is chosen for simulation. The indenter and the platform are set as discrete rigid. The platform is fixed and the indenter is given a forced displacement as experiment. A penalty based contact is set for the contact parts assuming a friction coefficient of 0.1 .

\subsection{Modeling and validation of the jellyroll}

In previous studies, the uniaxial stress-strain model for the compression of the jellyroll is expressed in two forms. The first one is expressed as Eq. (1), as suggested by Greve and Fehrenbach [13]:

$\sigma=A \varepsilon^{n}+B$ where $A$ and $B$ are the parameters to be determined, $\sigma$ is the stress, $\varepsilon$ is the strain, and $n$ is the hardening exponent. This model is actually reduced from the flow rule in the form of

$\sigma\left(\bar{\varepsilon}^{p}\right)=\left[\sigma_{\text {plateau }}-\left(\sigma_{\text {plateau }}-\sigma_{\text {yield }}\right) * \exp \left[-\frac{\bar{\varepsilon}^{p}}{\bar{\varepsilon}_{\text {ref }}}\right]\right]$

where $\sigma_{\text {yield }}$ is the initial yield stress, $\sigma_{\text {plateau }}$ is the intermediate plateau stress, $\bar{\varepsilon}_{\text {ref }}$ shapes the curvature of the hardening curve in between, $\bar{\varepsilon}^{p}$ is the plastic strain, and $\sigma\left(\bar{\varepsilon}^{p}\right)$ is the stress. The second form is suggested by Wierzbicki and Sahraei [15], who used the concept of a volumetric strain, and is expressed as

$\sigma=A \varepsilon^{n}$

where $A$ is the amplitude and $n$ is the exponent.

The abovementioned models actually share the same stressstrain relationship and are both capable of providing excellent predictions for the jellyroll in radial compression. The only 
difference for the two models is that the model in Eq. (1) may have a slightly better prediction because it describes the slow increase of stress in small strain simultaneously with a more rapid increase of stress in the larger strain domain owing to the "densification-like" behavior in jellyroll. Commercialized 18650 LIBs with $2250 \mathrm{mAh}$ nominal capacity, $4.2 \mathrm{~V}$ charge voltage, and $43.6 \mathrm{~g}$ weight are chosen in this study. The jellyroll of this cell is winded with two layers of the separator (polypropylene), one layer of negative electrode (graphite glued to the copper collector), and one layer of positive electrode $\left(\mathrm{LiCoO}_{2}\right.$ adhering to the aluminum collector). The thicknesses of the respective layers are $0.018,0.165$, and $0.159 \mathrm{~mm}$.

Thus, Eq. (1) is set as the calibration model to describe the uniaxial compression of the jellyroll in this study. To quantify the fitting parameters in Eq. (1), three repeated radial compression tests are conducted with $\mathrm{SOC}=0$ based on the INSTRON 5966 material testing platform at a loading rate of $5 \mathrm{~mm} / \mathrm{min}$ (nominal strain rate is $0.0046 \mathrm{~s}^{-1}$ ), which is regarded as quasi-static loading. Fig. 1(b) illustrates the force-displacement curve extracted from the tests.

A rather simple but useful method is employed to convert the load-displacement curve into the nominal stress-strain relationship. Nominal stress $\sigma_{n}$ can be considered as

$\sigma_{n}=\frac{F}{S_{c}}$

where $F$ is the load and $S_{c}$ refers to the contact area. $S_{c}$ may be estimated via

$S_{c}=l_{c} b_{c}$

where $l_{c}$ is the length of the cell, and the contact width $b_{c}$ can be further obtained as

$b_{c}=2 R \arccos \left(\frac{R-s / 2}{R}\right)$

where $R$ denotes the radius of the cell, and $s$ is the displacement of the indenter. Accordingly, the nominal strain $\varepsilon_{\text {norminal }}$ can be expressed as $\varepsilon_{n}=\frac{s}{2 R}$

The converted nominal stress-strain curve is shown in Fig. 1(c), and the parameters $A, B$, and $n$, which are found to be $930,3.4$, and 0.8 , respectively, can be fitted by this curve. The Young's modulus $E_{\text {jellyroll }}^{r}=500 \mathrm{MPa}$ is chosen for the jellyroll in the radius direction because of the plastic strain hardening curve, and a small Poisson's ratio $v_{\text {jellyroll }}=0.15$ is given according to previous open literature [13].

As shown in Fig. 1(e), the mechanical model of jellyroll $\sigma=930 \varepsilon^{0.8}+3.4$ can well present the mechanical behavior of LIB under compression, where the coefficient of determination $r^{2}=1-\sum_{i=1}^{n}\left(y_{i}-f_{i}\right)^{2} / \sum_{i=1}^{n}\left(y_{i}-\bar{y}\right)^{2}=0.99$ (where $y_{i}$ is the experiment data, $f_{i}$ is the simulation data or the fitting data and $\bar{y}$ is the mean of the experiment data). Neverthless, the performance is not acceptable for bending since isotropic model cannot provide an anisotropic mechanical response to the whole LIB during bending when the upper surface is in compression and the lower one is in tension status. In this case, an anisotropic model is necessary to overcome this challenge.

The engineering constants used for the anisotropic properties with the stress-strain constitutive law are shown as follows:

$$
\left\{\begin{array}{l}
\varepsilon_{11} \\
\varepsilon_{22} \\
\varepsilon_{33} \\
\gamma_{12} \\
\gamma_{23} \\
\gamma_{31}
\end{array}\right\}=\left[\begin{array}{cccccc}
\frac{1}{E_{11}} & -\frac{v_{21}}{E_{22}} & -\frac{v_{31}}{E_{33}} & 0 & 0 & 0 \\
-\frac{v_{12}}{E_{11}} & \frac{1}{E_{22}} & -\frac{v_{32}}{E_{33}} & 0 & 0 & 0 \\
-\frac{v_{13}}{E_{11}} & -\frac{v_{23}}{E_{22}} & \frac{1}{E_{33}} & 0 & 0 & 0 \\
0 & 0 & 0 & \frac{1}{G_{12}} & 0 & 0 \\
0 & 0 & 0 & 0 & \frac{1}{G_{23}} & 0 \\
0 & 0 & 0 & 0 & 0 & \frac{1}{G_{31}}
\end{array}\right\}\left\{\begin{array}{l}
\sigma_{11} \\
\sigma_{22} \\
\sigma_{33} \\
\tau_{12} \\
\tau_{23} \\
\tau_{31}
\end{array}\right\}
$$

where $E_{11}, E_{22}$, and $E_{33}$ are the Young's modulus in the $x_{1}, x_{2}$, and $x_{3}$ directions, respectively; $G_{12}, G_{23}$, and $G_{31}$ is the shear modulus of planes $x_{1}-x_{2}, x_{2}-x_{3}$, and $x_{3}-x_{1} ; v_{i j}$ is the Poisson's ratio; and subscripts " 1 ," "2," and " 3 " stand for the coordinate system.

To simplify the model, the $x_{1}-x_{2}$ plane is considered as isotropic, such that $E_{11}=E_{22}=E_{p}, \quad v_{31}=v_{32}=v_{t p}, \quad v_{13}=v_{23}=v_{p t}, \quad$ and

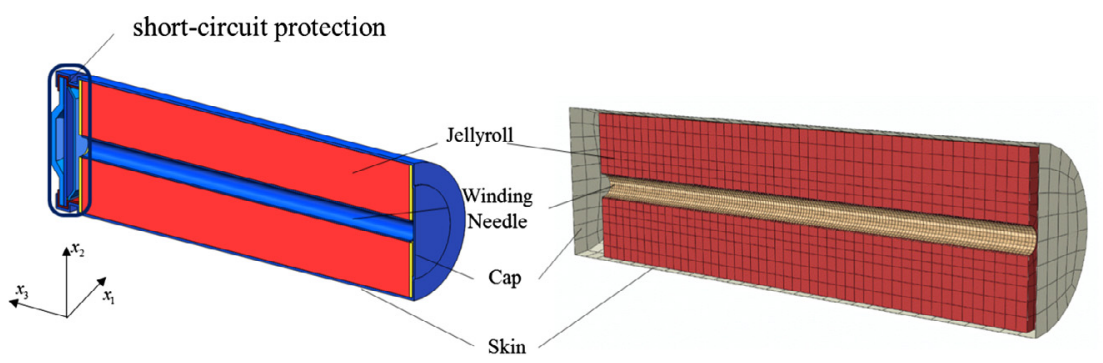

(a)

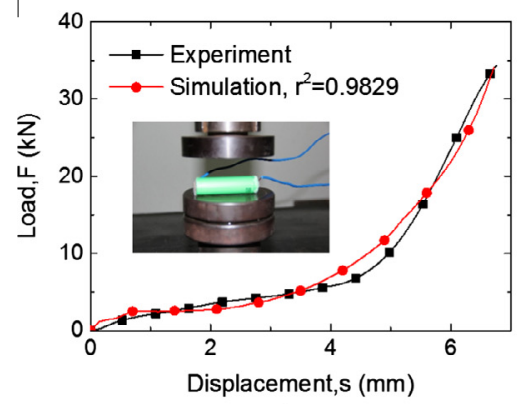

(b)

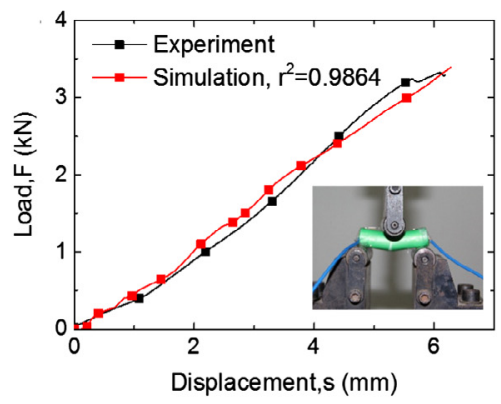

(c)

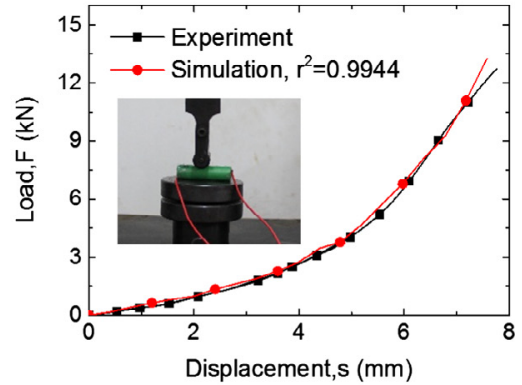

(d)

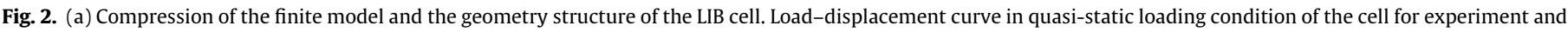
simulation: (b) radial compression, (c) bending, and (d) indentation. 


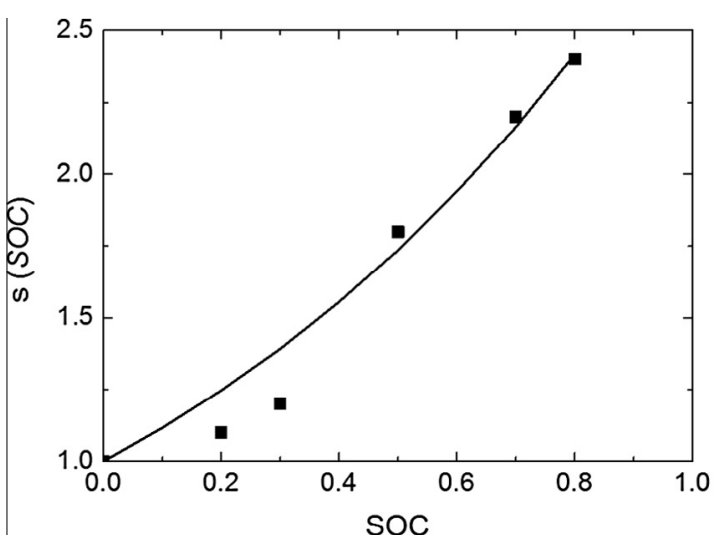

(a)

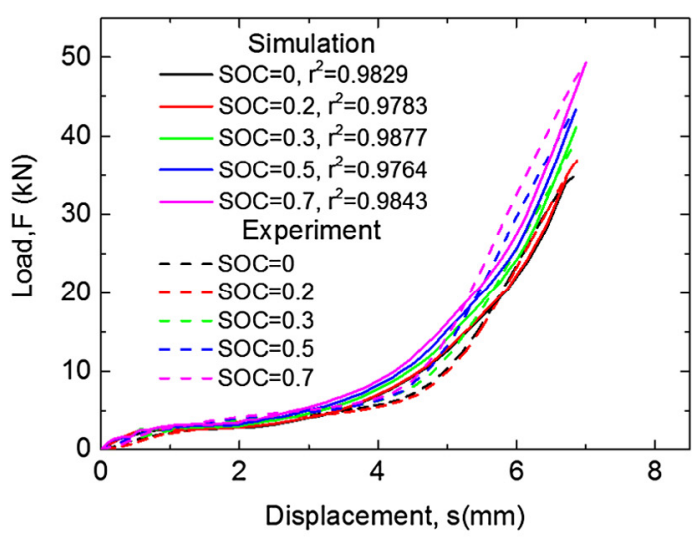

(b)

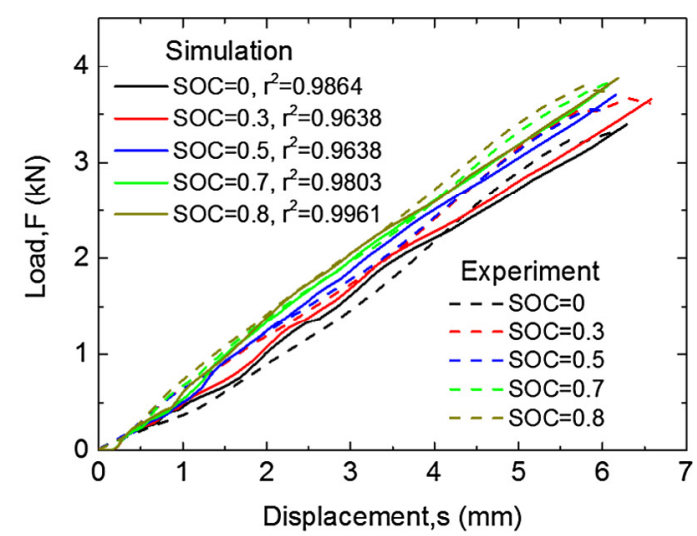

(c)

Fig. 3. (a) Fitting of the function relationship of $s(\mathrm{SOC})$. Comparison of the experiment and simulation using the SOC related model in (a) radial compression condition and (b) bending condition.

$G_{13}=G_{23}=G_{t}$, where $p$ and $t$ stand for "in plane" and "transverse" respectively. The stress-strain laws are then reduced to

$$
\left\{\begin{array}{l}
\varepsilon_{11} \\
\varepsilon_{22} \\
\varepsilon_{33} \\
\gamma_{12} \\
\gamma_{23} \\
\gamma_{31}
\end{array}\right\}=\left[\begin{array}{cccccc}
\frac{1}{E_{p}} & -\frac{v_{p}}{E_{p}} & -\frac{v_{t p}}{E_{t}} & 0 & 0 & 0 \\
-\frac{v_{p}}{E_{p}} & \frac{1}{E_{p}} & -\frac{v_{t p}}{E_{t}} & 0 & 0 & 0 \\
-\frac{v_{p t}}{E_{p}} & -\frac{v_{p t}}{E_{p}} & \frac{1}{E_{t}} & 0 & 0 & 0 \\
0 & 0 & 0 & \frac{1}{G_{p}} & 0 & 0 \\
0 & 0 & 0 & 0 & \frac{1}{G_{t}} & 0 \\
0 & 0 & 0 & 0 & 0 & \frac{1}{G_{t}}
\end{array}\right]\left\{\begin{array}{l}
\sigma_{11} \\
\sigma_{22} \\
\sigma_{33} \\
\tau_{12} \\
\tau_{23} \\
\tau_{31}
\end{array}\right\}
$$

where $v_{t p} / E_{t}=v_{p t} / E_{p}, G_{p}=E_{p} / 2\left(1+v_{p}\right)$, and only five independent constants are left. The in-plane Young's modulus $E_{p}$ is equal to the Young's modulus in the radial direction used in the isotropic model, $E_{p}=E_{\text {jellyroll }}^{r}=500 \mathrm{MPa}$. The transverse Young's modulus $E_{t}$ can be confirmed by a compression test in the $x_{3}$ direction. The elastic stage is before $1.2 \mathrm{~mm}$ because the separator is slightly longer than the electrode, $E_{t}=1500 \mathrm{MPa}$, as shown in Fig. 1(d). The "in-plane" Poisson's ratio $v_{p}$ is chosen as the Poisson's ratio of the isotropic model, where $v_{p}=v_{\text {jellyroll }}=0.15 . v_{p t}$ and $v_{t p}$ are estimated by the stability relationships as follows [32]:

$$
\left\{\begin{array}{l}
E_{p}, E_{t}, G_{p}, G_{t}>0 \\
\left|v_{p}\right|<1 \\
\left|v_{p t}\right|<\left(E_{p} / E_{t}\right)^{1 / 2} \\
\left|v_{t p}\right|<\left(E_{t} / E_{p}\right)^{1 / 2} \\
1-v_{p}^{2}-2 v_{t p} v_{p t}-2 v_{p} v_{t p} v_{p t}>0
\end{array}\right.
$$

Thus, $v_{p t}=0.1$ and $v_{t p}=0.3$ are confirmed. A small modulus $300 \mathrm{MPa}$ is chosen for the transverse shear modulus $G_{t}$, and the plane sheer modulus $G_{p}$ is calculated using the relationship as follows:

$$
G_{p}=E_{p} / 2\left(1+v_{p}\right)=217 \mathrm{MPa}
$$

The plasticity property also differs in three principal directions. In the $x_{1}-x_{2}$ plane, the plasticity properties are calculated by the compression tests, which are the same as the isotropic model:

$$
\sigma=930 \varepsilon^{3.4}+0.8
$$

The yield stress ratio $R=\left[\begin{array}{ccc}\frac{\bar{\sigma}_{11}}{\sigma_{0}} & \frac{\bar{\sigma}_{12}}{\tau_{0}} & \frac{\bar{\sigma}_{13}}{\tau_{0}} \\ & \frac{\bar{\sigma}_{22}}{\sigma_{0}} & \frac{\bar{\sigma}_{23}}{\tau_{0}} \\ & & \frac{\bar{\sigma}_{33}}{\sigma_{0}}\end{array}\right]$ is proposed to describe the anisotropic properties, where $\sigma_{0}$ is the reference yield stress set as $0.8 \mathrm{MPa}, \tau_{0}=\sigma_{0} / \sqrt{3}$, and $\bar{\sigma}_{i j}$ is the measured yield stress value. Owing to the isotropy in the $x_{1}-x_{2}$ plane, $\bar{\sigma}_{11}, \bar{\sigma}_{12}$, and $\bar{\sigma}_{22}$ can be calculated by the compression tests, the result of which is $0.8 \mathrm{MPa}$, and $\bar{\sigma}_{33}$ is set as the cut-off stress in tension tests, i.e., $10 \mathrm{MPa}$ in Ref. [14].

Using this model can well predict the mechanical properties of jellyroll in both bending and compression, as illustrated in Fig. 1(e), which shows the comparison of the isotropic and current anisotropic models. Clearly, both isotropic and anisotropic models can well predict the mechanical response of LIB during compression evidenced by $r^{2}=0.99$. On the other hand, the anisotropic model may well predict the bending experiment results with $r^{2}=0.81$, but the isotropic model fails to provide a good prediction. Therefore, the jellyroll bears a considerable amount of loading during bending, which cannot be regarded as trivial.

\subsection{Modeling and validation of the battery cell}

Based on the model established for jellyroll, one should also add the battery shell to describe the entire mechanical behavior of the 18650 battery cell. The 18650 LIB shell is mostly made of steel or aluminum to maintain the shape of the jellyroll. In the present study, the shell is made of common steel, with the mechanical properties obtained from Ref. [13]. Young's modulus is $E_{\text {shell }}=207 \mathrm{GPa}$, and Poisson's ratio is $v_{\text {shell }}=0.3$. The strain hardening can be well represented by the Johnson-Cook model, which is written as

$\sigma=\left(0.6+600 \bar{\varepsilon}^{p n}\right)\left(1+0.062 \ln \dot{\bar{\varepsilon}}^{p *}\right)$

where $\bar{\varepsilon}^{p}$ is the plastic strain, and $\dot{\bar{\varepsilon}}^{p *}=\frac{\bar{c}^{p}}{\frac{\bar{c}}{\bar{p}}}$ is the dimensionless plastic strain rate, with $\dot{\bar{\varepsilon}}_{0}^{p}=1 \mathrm{~s}^{-1}$ as a reference strain rate [29]. 


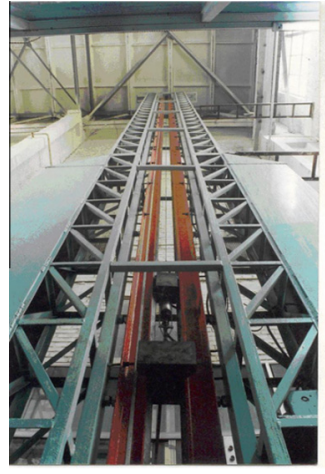

(a)

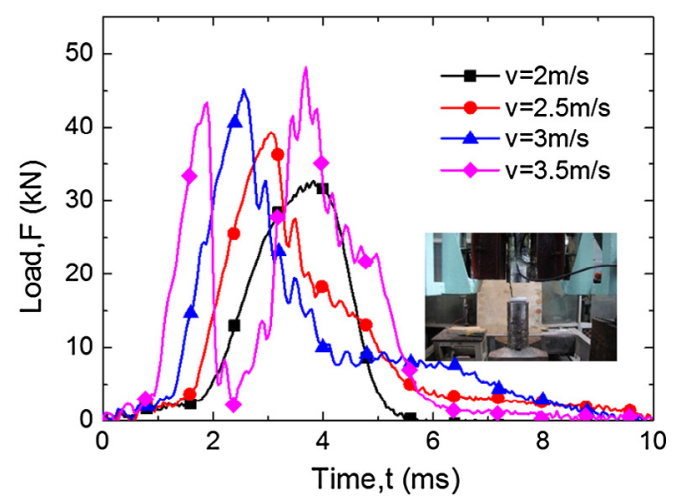

(b)

Fig. 4. (a) An upright view of the drop testing machine. (b) Load-time curve of the drop experiment.

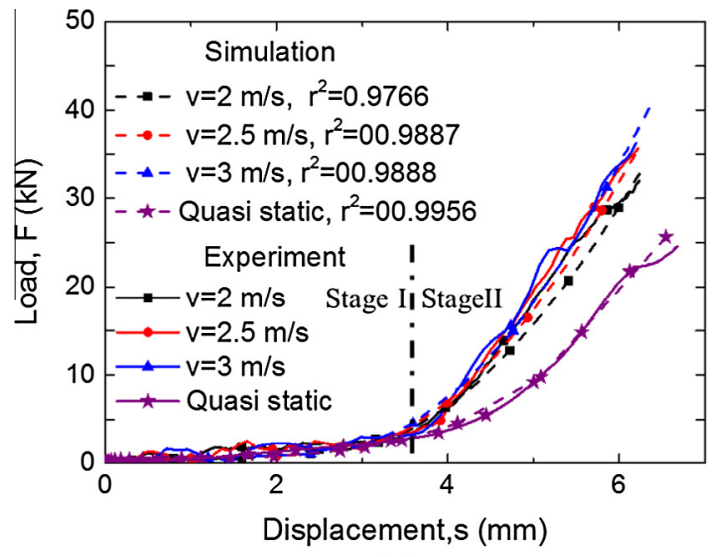

(a)

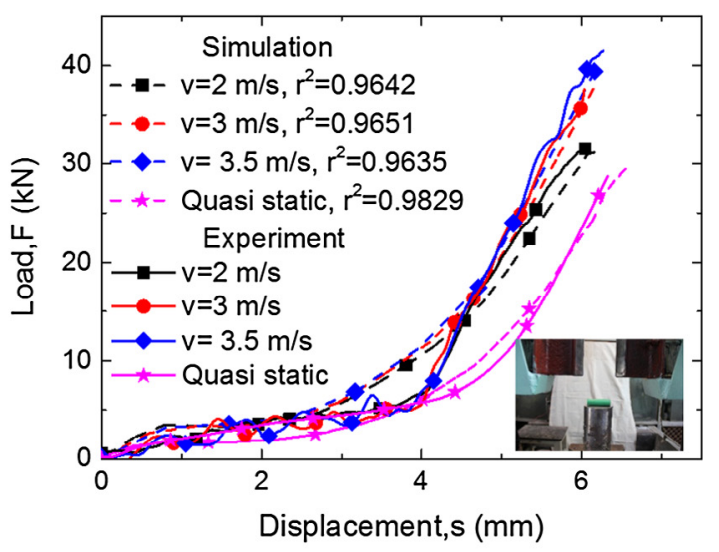

(b)

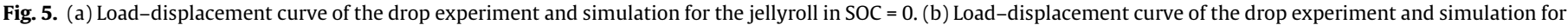
the LIB cell in $\mathrm{SOC}=0$.

The finite element model of the LIB cell is constructed strictly following the real-world cell, except for the removal of some tiny but geometrically complicated parts to facilitate meshing, computational convergence, and efficiency, as shown in Fig. 2(a). The cap and winding needle have the same material properties as the shell.

Simulations are run in parallel for comparison with the data obtained from experiments shown in Fig. 2(b)-(d) separately. Values of $r^{2}$ for the simulation results using the established mechanical model are all greater than 0.98 for indentation, compression and bending. Therefore, this model can well describe and predict the mechanical response of the LIB cell under radial compression, indentation, and bending.

\subsection{Inclusion of the SOC effect}

The reason behind the stiffening mechanism for the higher SOC value has been proven to be the Li-ion insertion in anode [31]. The reaction force of a fully charged cell is $40 \%$ larger than that of an empty cell in radial compression tests.

Through an analogy with the strain-rate effect, a possible hardening model is expressed as

$\sigma=f(\varepsilon, \mathrm{SOC})$

When $\mathrm{SOC}=0$, the hardening model is degenerated to Eq. (12). Eq. (14) can be reduced to a multiple relationship as follows:

$\sigma=s(\mathrm{SOC}) f(\varepsilon, 0)$ where $s(\mathrm{SOC})$ presents the function relationship with the value of SOC, which can be calculated by simulating $s(\mathrm{SOC})$ with multiple values. Fig. 3(a) presents the description of the SOC function, which may be expressed as

$s(\mathrm{SOC})=\exp (1.103 \times \mathrm{SOC})$

The compression simulation of this model and the experiment results are compared in Fig. 3(b). The simulation can have a good agreement with the experiment in each SOC value with $r^{2}=0.97-0.98$, indicating that the mechanical behavior can be predicted by this model with consideration of the SOC effect. In bending conditions, the mechanical properties related to SOC can also be well expressed by this model with $r^{2}=0.96-0.99$, as illustrated in Fig. 3(c).

\section{Numerical modeling of LIB in dynamic loadings}

\subsection{The experiment setups and simulation boundary conditions}

Drop-weight experiments shown in Fig. 4(a) are carried out to study the dynamic effect. The weight of the hammer is $31.6 \mathrm{~kg}$, according to the estimation of crash energy based on quasi-static loading. The initial impact velocities are set as $2,2.5,3$, and $3.5 \mathrm{~m} / \mathrm{s}$, representing the respective drop heights of 204,318 , 450 , and $625 \mathrm{~mm}$. The acceleration sensor is set on the hammer for measuring the acceleration of the hammer. ABAQUS/Explicit 


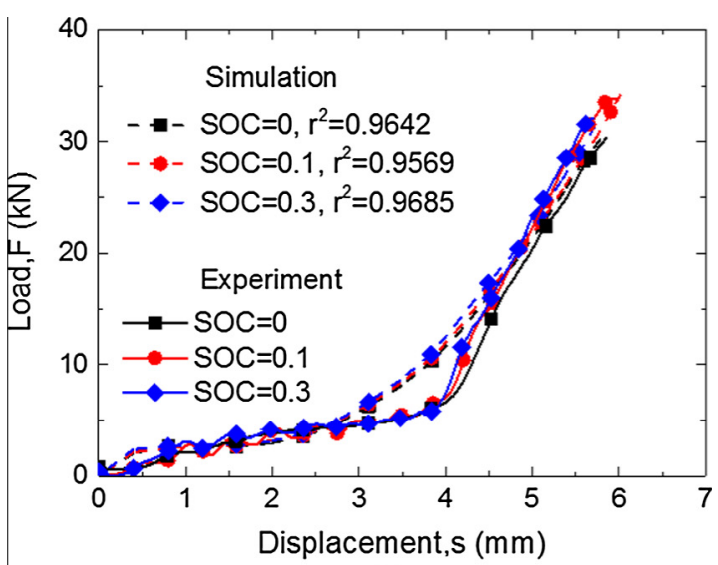

(a)

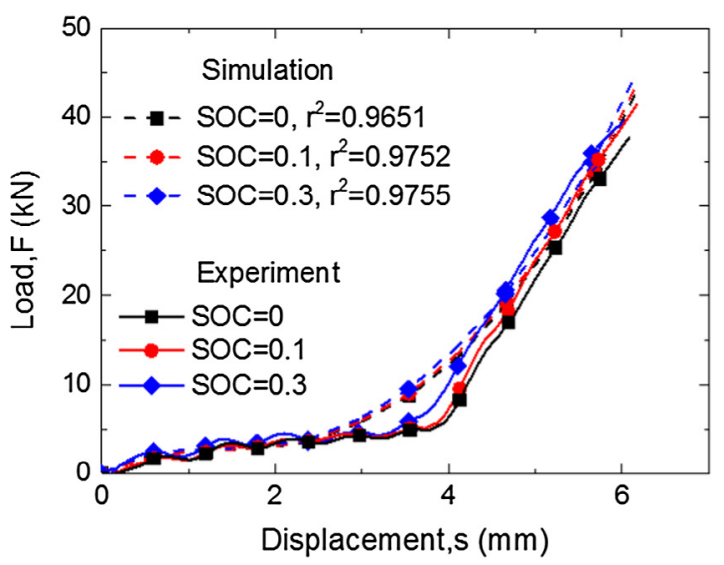

(b)

Fig. 6. Load-displacement curve of the drop experiment and simulation for the cell in various SOCs with velocity of (a) $2 \mathrm{~m} / \mathrm{s}$ and (b) $3 \mathrm{~m} / \mathrm{s}$.

is chosen for dynamic simulation study. The indenter and the bearing are set as discrete rigid. The bearing is fixed and the indenter is given an initial velocity as experiment. A penalty based contact is set for the contact parts assuming a friction coefficient of 0.1 .

\subsection{Modeling and validation of the jellyroll}

Fig. 4(b) shows the load-time curves extracted from the tests. Note that the force-time history curve has two peaks at the high drop-weight velocity (i.e., $3.5 \mathrm{~m} / \mathrm{s}$ ), while only one peak can be observed at low-velocity cases. The first peak indicates the fracture of the jellyroll, while the second one is the rebound of the drop hammer. The load-displacement curve can be easily translated from the load-time curve by $\int v_{\text {hammer }} d t$, as depicted in Fig. 5(a).

The diameter of the hollow winding needle is approximately $3 \mathrm{~mm}$ in diameter by measurement. During deformation, this hollow hole would collapse first with little strain-rate effect. Theoretically, the structure of the whole cell has little dynamic effect before the critical nominal strain $\varepsilon_{c} \approx 0.2$. Thus, the jellyroll is regarded as strain-rate independent before the critical nominal strain, but highly strain-rate dependent after the threshold strain, such that the dynamic model would be suggested in the form of a two-stage description. Stage I is mainly for eliminating gaps of the jellyroll, and so the jellyroll has little dynamic effect in this stage. Stage II begins with the densification of the jellyroll when most of the jellyroll materials are involved, such that the dynamic effect is obvious in this stage.
The rate-dependent model can be written as

$\sigma=f(\varepsilon, \dot{\varepsilon})$

when $\varepsilon<\varepsilon_{c}$, the function can be written as Eq. (12); when $\varepsilon \geqslant \varepsilon_{c}$, the hardening stress is equal to the following:

$\sigma=\sigma_{c}+B\left(\varepsilon^{n}-\varepsilon_{c}^{n}\right) g(\dot{\varepsilon})$

where $n$ is the hardening indicator equal to 3.4, which comes from Eq. (12), and $\sigma_{c}$ is the critical stress set as

$\sigma_{c}=0.8+930 \varepsilon_{c}^{3.4}$

$g(\dot{\varepsilon})$ is the strain-rate dependent function fit by the drop-weight experiment. After entering the densification regime, the jellyroll is regarded as a homogeneous solid material and the strain-rate dependent material is expressed as

$g(\dot{\varepsilon})=\exp \left(0.02085 \dot{\varepsilon}^{*}\right)$

where $\dot{\varepsilon}^{*}$ equals $\dot{\varepsilon} / \dot{\varepsilon}^{\text {ref }}$, and $\dot{\varepsilon}^{\text {ref }}$ is the reference strain rate set as $1 \mathrm{~s}^{-1}$.

This model is used in finite element simulation with the comparison of drop-weight test results of 2 and $3 \mathrm{~m} / \mathrm{s}$, as shown in Fig. 5(a). A good agreement between numerical simulation and experiment results for the jellyroll only can be expected in dynamic crushing, where $r^{2}=0.98-0.99$.

\subsection{Validation of the battery cell}

With the strain-rate dependent descriptions for both jellyroll and battery shell already established, one may further verify the dynamic mechanical response of the two parts combined. Here, impact velocities of 2,3 , and $3.5 \mathrm{~m} / \mathrm{s}$ are proposed for the experiment and simulation, and the results are shown in Fig. 5(b). The model is confirmed to predict the crush accurately with $r^{2}=0.96-0.98$. However, a deviation occurs in the densification stage amplified from the slight deviation in the static model mainly generated from the simple battery shell model.

The cells are usually well protected in crash safety design and will not deform over the critical strain $\varepsilon_{c}$ in small-size EV crash tests. These results indicate that the quasi-static model of the jellyroll and battery shell can be used in most vehicle crash situations. However, the crash velocity of the standard is too low in comparison with that in actual accidents, and the protection of the cell limits the arrangement of the vehicle. Thus, this dynamic mechanical model of the jellyroll and battery shell can be used in the crash safety design of high vehicle velocity and the new EV arrangement plan.

\subsection{SOC inclusion}

The above study presents a model that can predict the mechanical properties in various SOC values and at different crash velocities. Going one step further, the model must be related with the SOC value, which may be expressed as

$\sigma=f(\varepsilon, \operatorname{SOC}, \dot{\varepsilon})$

According to Eqs. (15) and (18), $\varepsilon$, SOC, and $\dot{\varepsilon}$ are independent parameters, such that the stress-strain curves can be represented as

$\sigma=\left(A \varepsilon^{n}+B\right) s(\mathrm{SOC}) g(\dot{\varepsilon}) k(\dot{\varepsilon}, \mathrm{SOC})$

where $k(\dot{\varepsilon}, \mathrm{SOC})$ is the correlation function, which may express the relationship of strain rate and SOC. This paper predict it as

$k(\dot{\varepsilon}, \mathrm{SOC})=1$

Then, the equation can be written as 


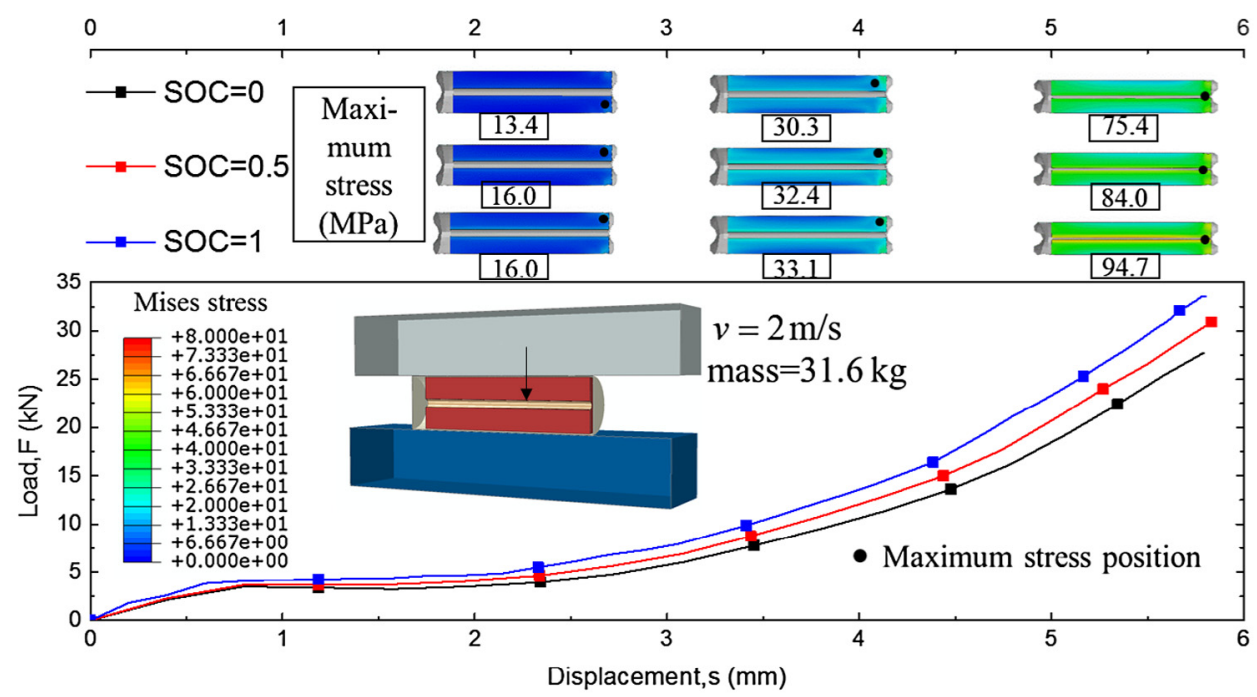

(a)

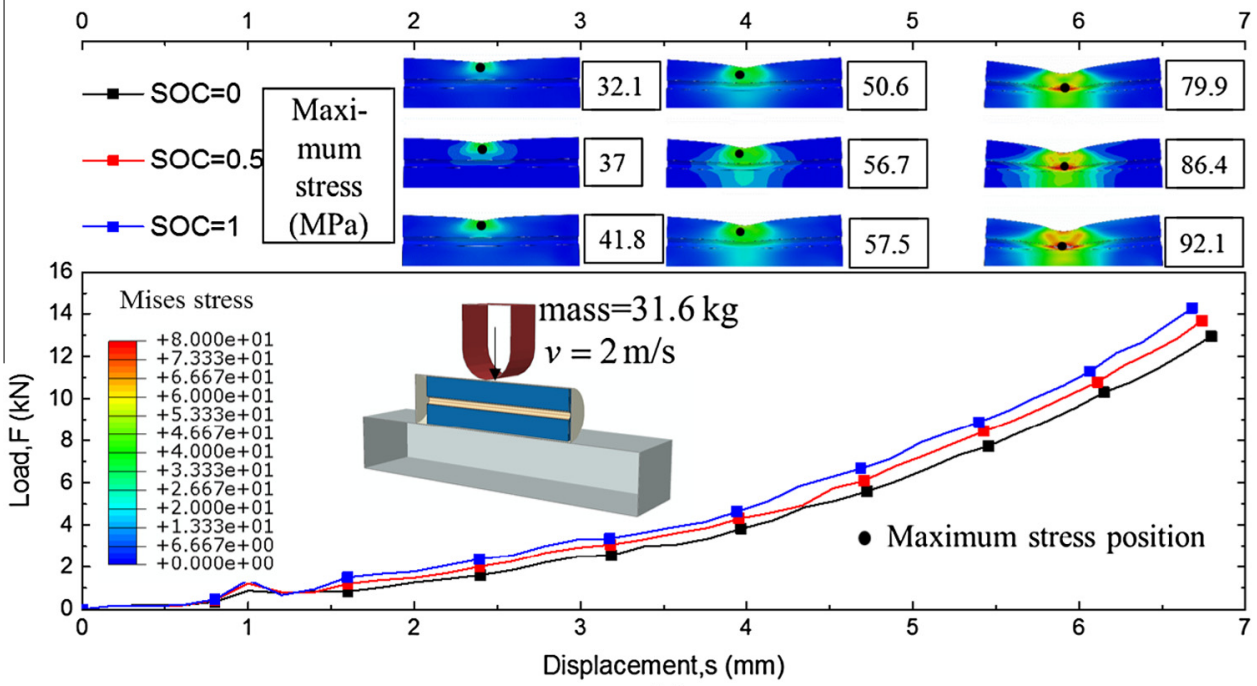

(b)

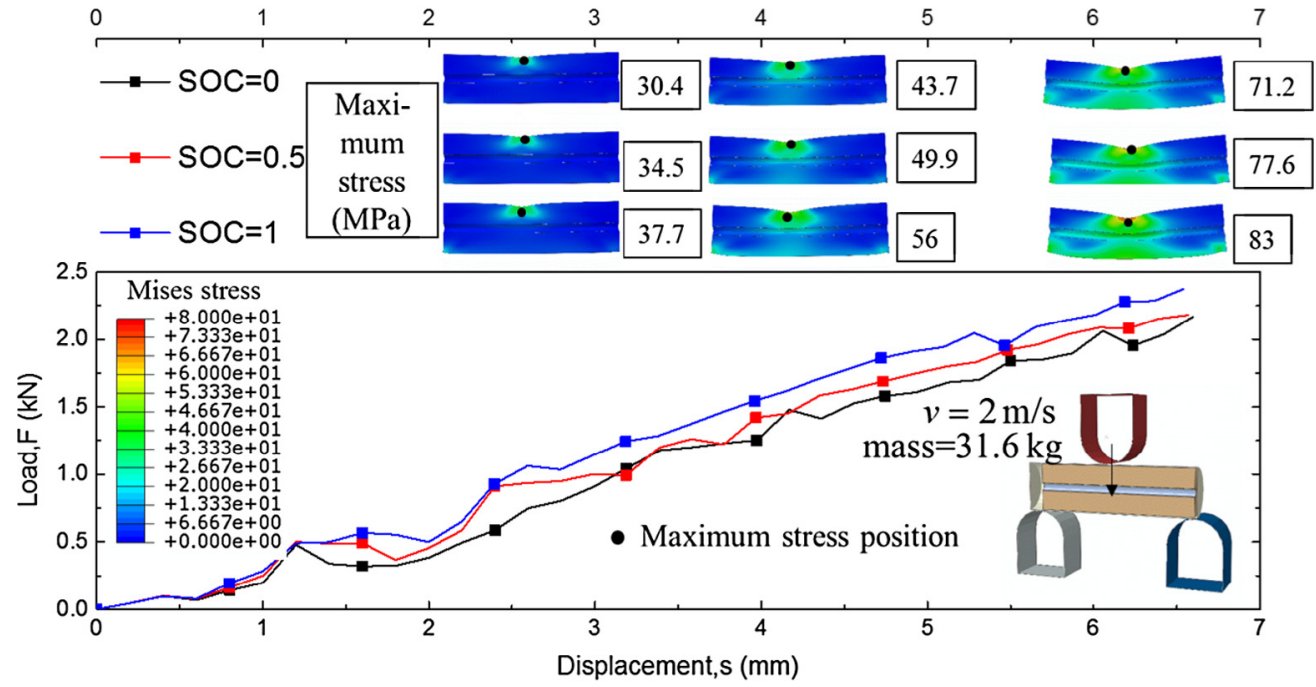

(c)

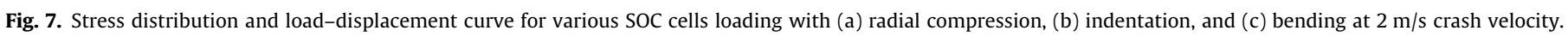




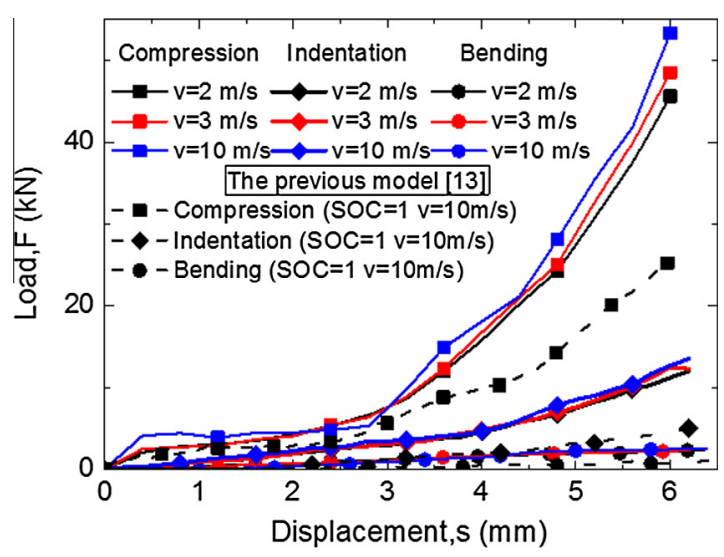

Fig. 8. Load-displacement curve of the simulation for the fully charged cells in bending, radial compression, and indentations with various SOC values.

$\sigma= \begin{cases}\left(930 \varepsilon^{3.4}+0.8\right) \mathrm{e}^{1.10350 c}, & \varepsilon \leqslant \varepsilon_{c} \\ {\left[\sigma_{c}+930\left(\varepsilon^{3.4}-\varepsilon_{c}^{3.4}\right) \mathrm{e}^{1.10350 c}\right] \mathrm{e}^{0.02085 \dot{\varepsilon}^{*}},} & \varepsilon>\varepsilon_{c}\end{cases}$

The established strain rate and SOC effect coupled model can be validated by experiment data for $2 \mathrm{~m} / \mathrm{s}$ impact velocity at SOC 0 , 0.1 , and 0.3 , as well as $3 \mathrm{~m} / \mathrm{s}$ impact velocity at SOC $0,0.1$, and 0.3. A good agreement can be observed among the simulation and experiment results shown in Fig. 6(a) and (b), proving the validity of the coupled model, where $r^{2}=0.96-0.98$.

\section{Discussions}

In this section, with the help of the established finite element model, the potentially highly dangerous experiment situations that LIB may undergo during extreme mechanical loadings are investigated.

\subsection{Various SOC values at dynamic loadings}

Battery cells in different SOCs express different mechanical properties in crushing conditions. Simulations for cells in various SOCs are carried out at bending, radial compression, and indentations, where the impact velocity is set as $2 \mathrm{~m} / \mathrm{s}$.

In compression conditions, the reaction force of the higher SOC cells is larger than that of the lower ones, as shown in Fig. 7(a). The stress distribution of the jellyroll is also slightly different in the same deformation in various SOCs, and the maximum stress of the jellyroll is changed due to the SOC, e.g., the maximum stress of a fully charged jellyroll will be about $10-25 \%$ larger than those of empty ones.

The result of indentation and bending conditions are shown in Fig. 7(b) and (c), wherein the reaction force and maximum stress of the high SOC are also higher in the same load displacement. Researchers have built failure criterions from stress state to predict the initial short of the cell $[13,29]$. Cells at higher SOC will reach to a larger stress condition under the same deformation (displacement), illustrated in Fig. 7. According to the established mechanical integrity criterion from stress state to predict the initial short of the cell in Ref. [10], cells at higher SOC should fail (or become internally short circuited) in a smaller displacement which confirms that higher charged battery should be more dangerous during crash situation from the mechanical perspective. The maximum stress positions are close to the indentor at each SOC, and the values are $10-30 \%$ and $15-30 \%$ larger in fully charged cells than the empty ones. The change in stress distribution of the cells in various
SOCs means the mechanical integrity failure of the LIB cells are also SOC dependent.

\subsection{Various loading speeds for high SOC value}

As indicated in Section 4.1, crush experiments are dangerous for high SOC cells. Numerical simulations, however, can easily predict the mechanical behavior by using the suggested model without any hazards. Fig. 8 shows the load-displacement curve of the fully charged cell in radial compression, indentation, and bending crash situations. Owing to the strain-rate dependency of the jellyroll, the reaction force is larger in high-speed impact in all three loading conditions, and the change in reaction force are $18 \%, 9.7 \%$, and $11.2 \%$ in radial compression, indentation, and bending, respectively, from $2 \mathrm{~m} / \mathrm{s}$ to $10 \mathrm{~m} / \mathrm{s}$. A comparison with the empty cell in quasi-static conditions reveals that this reaction force is increased by about $200 \%, 170 \%$, and $135 \%$, respectively. Fig. 8 also demonstrates that using the isotropic model may underestimate the prediction of mechanical behavior of the LIB subject to dynamic loading, such that the model 18650 LIB with coupled strain rate and SOC dependencies is necessary to ensure a crashworthy LIB design.

\section{Concluding remarks}

Correctly predicting the mechanical behaviors of LIBs subject to mechanical loading is critical to understanding the mechanical integrity of LIBs and ensuring LIB crash safety. To improve the available isotropic LIB model in open literature, this paper established an anisotropic model that can describe the mechanical response of the LIB at bending, indentation, and compression loadings. Additionally, the SOC dependency of the LIB in terms of mechanical behavior was considered in the numerical model. With the strain effects of the jellyroll and the battery shell taken into consideration, a dynamic mechanical model for LIB that will enable the wider application of the mechanical model was suggested. A satisfactory agreement among numerical simulation and experiment results were observed. Finally, SOC-dependent dynamic behaviors and the mechanical stress status of the LIB at hazardous SOC values and high impact speeds were further discussed based on the model. The new mechanical model for computation is established with the full consideration of dynamic loading effect and SOC effect from the LIB mechanical integrity point of view. This new model may have improved 200\%, 170\%, and 135\% accuracy in compression, indentation and bending loading conditions separately compared with the original models in extreme situations. And this general mechanical model may lay a solid foundation for future research on LIB mechanical integrity and safety design.

\section{Acknowledgements}

This work is financially supported by the Fundamental Research Funds for the Central Universities, Beihang University, the startup fund for "Zhuoyue 100" titled professor, Beihang University, the National Natural Science Foundation of China under Grant numbers (11102017 and 11032001), Opening fund of State Key Laboratory of Nonlinear Mechanics, Institute of Mechanics, Chinese Academy of Sciences and Opening fund of State Key Laboratory for Strength and Vibration of Mechanical Structures, Xi'an Jiaotong University.

\section{References}

[1] Chan CK, Peng HL, Liu G, Mcllwrath K, Zhang XF, Huggins RA, et al. Highperformance lithium battery anodes using silicon nanowires. Nat Nanotechno 2008;3:31-5. 
[2] Cheng FY, Liang J, Tao ZL, Chen J. Functional materials for rechargeable batteries. Adv Mater 2011;23:1695-715.

[3] Goodenough JB, Kim Y. Challenges for rechargeable Li batteries. Chem Mater 2010;22:587-603.

[4] Kang B, Ceder G. Battery materials for ultrafast charging and discharging. Nature 2009;458:190-3.

[5] Sun Y-K, Myung S-T, Park B-C, Prakash J, Belharouak I, Amine K. High-energy cathode material for long-life and safe lithium batteries. Nat Mater 2009;8:320-4.

[6] Ovrum E, Bergh TF. Modelling lithium-ion battery hybrid ship crane operation. Appl Energy 2015;152:162-72.

[7] Vikstrom H, Davidsson S, Hook M. Lithium availability and future production outlooks. Appl Energy 2013;110:252-66.

[8] Zhou Y, Wang M, Hao H, Johnson L, Wang HW, Hao H. Plug-in electric vehicle market penetration and incentives: a global review. Mitig Adapt Strateg Glob Chang 2015;20:777-95.

[9] Chen J, Sun T, Qi Y, Li X. A coupled penetration-tension method for evaluating the reliability of battery separators. ECS Electrochem Lett 2014;3.

[10] Sahraei E, Kahn M, Meier J. Wierzbicki T. Modelling of cracks developed in lithium-ion cells under mechanical loading. RSC Adv 2015;5:80369-80.

[11] Chen J, Yan Y, Sun T, Qi Y, Li X. Deformation and fracture behaviors of microporous polymer separators for lithium ion batteries. RSC Adv 2014;4:14904-14

[12] Chen J, Yan Y, Sun T, Qi Y, Li X. Probing the roles of polymeric separators in lithium-ion battery capacity fade at elevated temperatures. J Electrochem Soc 2014;161:A1241-6.

[13] Greve L, Fehrenbach C. Mechanical testing and macro-mechanical finite element simulation of the deformation, fracture, and short circuit initiation of cylindrical lithium ion battery cells. J Power Sour 2012;214:377-85.

[14] Sahraei E, Campbell J, Wierzbicki T. Modeling and short circuit detection of 18650 Li-ion cells under mechanical abuse conditions. J Power Sour 2012;220:360-72.

[15] Wierzbicki T, Sahraei E. Homogenized mechanical properties for the jellyroll of cylindrical lithium-ion cells. J Power Sour 2013;241:467-76.

[16] Lai WI, Ali MY, Pan J. Mechanical behavior of representative volume elements of lithium-ion battery modules under various loading conditions. J Power Sour 2014;248:789-808.

[17] Lai W-I, Ali MY, Pan J. Mechanical behavior of representative volume elements of lithium-ion battery cells under compressive loading conditions. J Power Sour 2014;245:609-23.
[18] Belov D, Yang M-H. Failure mechanism of Li-ion battery at overcharge conditions. J Solid State Electrochem 2007;12:885-94.

[19] Yan S, Xiao X, Huang X, Li X, Qi Y. Unveiling the environment-dependent mechanical properties of porous polypropylene separators. Polymer 2014;55:6282-92.

[20] Jalkanen K, Karppinen J, Skogstrom L, Laurila T, Nisula M, Vuorilehto K. Cycle aging of commercial NMC/graphite pouch cells at different temperatures. Appl Energy 2015;154:160-72.

[21] Ping P, Wang Q, Huang P, Sun J, Chen C. Thermal behaviour analysis of lithiumion battery at elevated temperature using deconvolution method. Appl Energy 2014;129:261-73.

[22] Sheidaei A, Xiao XR, Huang XS, Hitt J. Mechanical behavior of a battery separator in electrolyte solutions. J Power Sour 2011;196:8728-34.

[23] Feng X, Weng C, Ouyang M, Sun J. Online internal short circuit detection for a large format lithium ion battery. Appl Energy 2016;161:168-80.

[24] Cannarella J, Liu XY, Leng CZ, Sinko PD, Gor GY, Arnold CB. Mechanical properties of a battery separator under compression and tension. J Electrochem Soc 2014;161:F3117-22.

[25] Chen JC, Yan YD, Sun T, Oi Y, Li XD. Deformation and fracture behaviors of microporous polymer separators for lithium ion batteries. RSC Adv 2014;4:14904-14.

[26] Sheidaei A, Xiao X, Huang X, Wang J. Mechanical characterization of a lithium ion battery separator using a dynamic mechanical analyzer. SAE techni paper. Detroit, MI; 2011.

[27] Sethuraman VA, Chon MJ, Shimshak M, Van Winkle N, Guduru PR. In situ measurement of biaxial modulus of $\mathrm{Si}$ anode for Li-ion batteries. Electrochem Commun 2010;12:1614-7.

[28] Amanieu H-Y, Aramfard M, Rosato D, Batista L, Rabe U, Lupascu DC. Mechanical properties of commercial $\mathrm{Mn}_{2} \mathrm{O}_{4}$ cathode under different states of charge. Acta Mater 2015;89:153-62.

[29] Xu J, Liu B, Wang L, Shang S. Dynamic mechanical integrity of cylindrical lithium-ion battery cell upon crushing. Eng Fail Anal 2015;53:97-110.

[30] Tao X, Du J, Sun Y, Zhou S, Xia Y, Huang $H$. Exploring the energy storage mechanism of high performance $\mathrm{MnO}_{2}$ electrochemical capacitor electrodes: an in situ atomic force microscopy study in aqueous electrolyte. Adv Funct Mater 2013;23:4745-51.

[31] Xu J, Liu B, Hu D. State of charge dependent mechanical integrity behavior of 18650 lithium-ion batteries. Sci Rep 2016;6:21829.

[32] Timoshenko SP, Goodier JN. Theory of elasticity. McGraw Hill Higher Education; 1970 